\title{
FAINT HII REGIONS IN THE MAGELLANIC CLOUDS
}

\author{
ERIC M. WILCOTS, PAUL HODGE \\ Department of Astronomy \\ University of Washington \\ Seattle, WA 98195 \\ USA
}

\begin{abstract}
We present CCD observations of several faint regions of active star formation in the Magellanic Clouds. For the five regions for which we were able to obtain photometric data we have extended the luminosity function (LF) two orders of magnitude below the previous faint limit of $10^{37} \mathrm{erg}$ $\mathrm{s}^{-1}$ (1). Our preliminary results indicate that the LF turns over at $10^{36} \mathrm{erg} \mathrm{s}^{-1}$ for the LMC and $10^{36.5}$ $\mathrm{erg} \mathrm{s}^{-1}$ for the SMC. It is also apparent that much of the H $\alpha$ emission fainter than $10^{36} \mathrm{erg} \mathrm{s}^{-1}$ is diffuse or filamentary in nature.
\end{abstract}

\section{The data and the luminosity function}

This presentation represents the preliminary results of a six-night observing run with the CTIO 0.9m telescope in November 1989. Unfortunately, since only two of the nights were photometric, we were able to obtain photometric data for only five regions - three in the LMC and two in the SMC. Each region was selected to be representative of the Clouds, although we selected against areas with bright, well-known $\mathrm{H}$ II regions.

Individual $\mathrm{H}$ II regions were identified visually and then integrated to obtain their apparent fluxes. Each region is classified roughly following the system established in $(2,3)$. Faint compact regions are those which look like traditional $\mathrm{H}$ II regions and contain candidate exciting stars. Much of the emission at these levels is associated with loops and filaments, or diffuse background emission. The diffuse regions are seemingly not associated with any particular exciting star and have very low surface brightnesses over their entire area. With a scale of 0.445 "/pixel the typical region we can identify in this study has an area of, at most, a few times $10^{3}$ square arcseconds, an order of magnitude smaller than the typical regions identified in (1).

The LF was obtained by first correcting the apparent fluxes for Galactic foreground extinction (no correction was made for internal extinction) and scaling to the distance to the Clouds (50kpc for the LMC and 70 for the SMC). The LFs are shown in Figures 1 and 2 for the LMC and SMC respectively, and are normalized that to a value of one at a flux of $10^{38} \mathrm{erg} \mathrm{s}^{-1}$ following (1). The previously published points are plotted as open squares with the new results from this work platted as filled squares. We are limited by small number statistics at the very faint levels, largely because we were unable to complete the observations of our targets. The error bars are derived from Poisson statistics.

Despite this limitation, the preliminary results show that the LF turns over at $10^{36} \mathrm{erg} \mathrm{s}^{-1}$ for LMC and at $10^{36.5} \mathrm{erg} \mathrm{s}^{-1}$ for the SMC. This latter result matches well with (1). For comparison, the LF for the Local Group galaxies GR8 and NGC 6822 turnover at about $10^{35.5}$ and $10^{36} \mathrm{erg} \mathrm{s}^{-1}$ respectively (3). The faintest discernible regions we are able to identify have fluxes of a few times 
$10^{35} \mathrm{erg} \mathrm{s}^{-1}$ even though we can detect $\mathrm{H} \alpha$ emission down to $10^{33} \mathrm{erg} \mathrm{s}^{-1}$. It is likely that there are very few if any individual $\mathrm{H}$ II regions with integrated fluxes lower than $10^{35} \mathrm{erg} \mathrm{s}^{-1}$.

In many regions there is a diffuse background emission at $\sim 10^{33} \mathrm{erg} \mathrm{s}^{-1}$ per square arcsecond. This diffuse emission does not seem to be associated with any particular exciting star. We are also finding that much of the faint emission is due to knotty, filamentary structure in the interstellar gas. How this structure reflects the density distribution of the interstellar medium has yet to be determined. While some of this complex structure does seem to be associated with possible exciting stars, much of it does not. Such structure in more distant galaxies is likely to be indistinguishable from more traditional $\mathrm{H}$ II regions.

\section{Conclusions}

This work represents the preliminary steps in a larger effort to understand more completely the nature of faint star-forming regions in the Magellanic Clouds. We are limited in what we can say here by the fact that we were only able to observe five of our fifteen targeted regions. The initial results presented here suggest that: 1) the LF for the Small and Large Magellanic Clouds turn over at $\sim 10^{36} \mathrm{erg} \mathrm{s}^{-1}$ with a faint limit of $\sim 10^{35} \mathrm{erg} \mathrm{s}^{-1} ; 2$ ) much of the emission fainter than $10^{36} \mathrm{erg} \mathrm{s}^{-1}$ is a mixture of diffuse and filamentary emission that may or may not be directly associated with exciting stars. Some of this emission may reflect the density structure of the interstellar medium; 3) the discemible regions of emission at these faint levels are likely to be an order of magnitude smaller in size than the faintest regions identified by (1). As shown in the case of DEM283c, high resolution observations reveal a complex, composite nature of what had been classified as a single H II region.

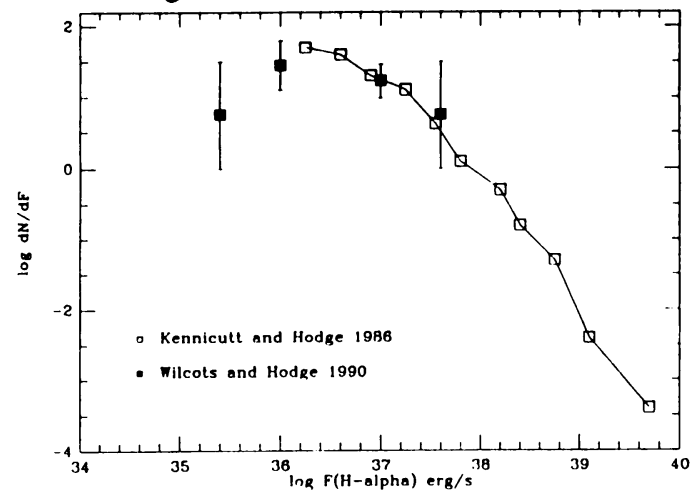

Figure 1. LMC H-alpha Luminosity Function

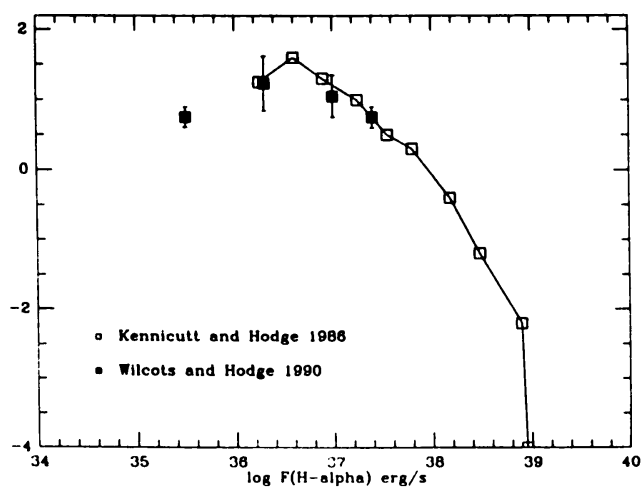

Figure 2. SMC H-alpha Luminosity Function

\section{Acknowledgments}

The authors would like to thank Dr. M.G. Lee, Nick Strobel and Drew Phillips for their help with the reduction and calibration. E.M. Wilcots was kindly supported by NASA Grant NGT-70050.

\section{References}

(1) Kennicutt, R.C. and Hodge, P.W. (1986), Ap. J. 306, 130.

(2) Hodge, P., Lee, M.G. and Kennicutt, R.C. (1989), P.A.S.P. 101, 640.

(3) Hodge, P., Lee, M.G. and Kennicutt, R.C. (1989), P.A.S.P. 101, 32. 